\title{
Using Behavioral Modeling and Simulation for Learning Communication Circuits and Systems
}

\author{
José M. de la Rosa \\ Institute of Microelectronics of Sevilla, IMSE-CNM (CSIC/Universidad de Sevilla) \\ C/ Américo Vespucio, Parque Tecnológico de la Cartuja, 41092 Sevilla, SPAIN \\ E-mail: jrosa@imse-cnm.csic.es
}

\begin{abstract}
This paper analyzes the use of behavioral simulation techniques to enhance the teaching-learning process in electrical engineering courses, specifically those dealing with circuits for communication systems. The method - which can be applied to both undergraduate and master courses - allows students to better understand complex circuit- and device-level phenomena, by describing them at a higher abstraction level. As a demonstration vehicle of the presented methodology, two examples are considered in this work: an analog front-end of a direct-conversion digital radio receiver and a $\Sigma \Delta$ modulator. In both cases, behavioral models of the different subcircuits have been implemented in MATLAB/SIMULINK and used by the students enrolled in two different courses: an undergraduate course and a master course. The results presented in this paper reveal that students become highly motivated and satisfied with the course contents and the proposed simulation-based learning methodology.
\end{abstract}

Keywords: behavioral modeling; simulation-based education.

\section{INTRODUCTION}

Simulation is an essential tool for the analysis and verification of integrated circuits. In the case of analog circuits, SPICE-like simulators have been traditionally used to evaluate their performance at different stages of the design procedure. However, the increasing complexity of analog systems - for instance in telecom applications - yield excessively long simulation CPU times. To cope with this problem, different alternative simulation techniques have been developed which improve the computational efficiency at the price of reducing the accuracy in their circuit-element models. Among others, one of the best accuracy-speed trade-offs is achieved by the socalled behavioral simulation approach. In this approach, a given circuit is partitioned into a set of sub-circuits - often called building blocks - modeled by a set of equations referred to as behavioral laws or behavioral models. This way, the accuracy of the simulation depends on how precisely these behavioral models emulate their actual operation [1].

From an educational perspective, the benefits of teaching behavioral modeling and simulation are twofold. On the one hand, students learn practical skills, which are essential for their professional career. On the other hand, they get a better insight about design problems in complex circuits and systems, because they deal with design parameters, which are much closer to the system-level specifications than transistor sizes and biasing, whose impact on the overall performance metrics is sometimes quite difficult to understand [2].

This work has been supported in part by the Spanish Ministry of Science and Innovation (with support from the European Regional Development Fund) under contracts TEC2007-67247-C02-01/MIC, TEC2010-14825/MIC, in part by the Consejería de Innovación, Ciencia y Empresa, under contract TIC-2532 and in part by the I Plan Propio de Docencia de la U. de Sevilla, LabCMA2010 project.
This work contributes to this topic and proposes the use of behavioral models as an effective pedagogical tool to enhance the quality of teaching and learning in electrical engineering, and particularly in those courses focused on analog circuits for communications. Two case studies are considered. The first one focuses on how to teach students to design Analog-toDigital Converters (ADCs) by means of behavioral simulation. The second one shows how to learn the system planning process of Radio Frequency (RF) front-ends in wireless transceivers. In both cases, behavioral models are implemented in MATLAB/SIMULINK, thus benefiting from its friendly user interface, powerful signal processing capability and high flexibility to incorporate new models. The teaching strategies presented in this paper - based on the experiences the author have had in the last ten years while teaching both undergraduate and master degree courses - demonstrate that the proposed simulation-based educational method help electrical engineering students comprehend the course material, being an efficient complement to traditional lectures presentations and lab exercises.

\section{CONTENTS AND OBJECTIVES OF THE COURSES}

The simulation-based educational methodology described in this paper has been implemented in the following courses:

- Electronic Circuits for Communications (EC), which is an undergraduate course given in two different bachelor programs at the University of Seville: an Electrical and Electronic Engineering degree and a Telecommunications Engineering degree. In both cases, the subjects covered in the course are focused on the design of analog, mixed-signal and RF integrated circuits for communications, putting emphasis on the design issues at building-block level, as well as their influence at system level.

- Wireless Transceivers: Standards, Techniques and Architectures (WT), which belongs to a master degree in Microelectronics, and covers different topics mainly focused on the transceiver architecture level of wireless communication systems.

Both courses are given in a 15-week class period, and correspond to six European Credit Transfer System (ECTS) credits - three of them being theoretical ECTS credits and the other three ones corresponding to practical ECTS credits. The contents of both courses are summarized below. 


\section{A. Undergraduate Course}

The EC course aims at teaching fundamental principles and practical design issues dealing with integrated circuits included in the analog front-ends of main telecom systems. The operation of these circuits is analyzed considering that they are part of a more complex system. Therefore, it is important to study not only their stand-alone operation but also the impact of their circuit non-ideal effects on the performance of entire telecom systems. From this perspective, it is important to provide students with suited tools to analyze the propagation of circuit error mechanisms through the different hierarchical levels of telecom systems under study.

Bearing this general learning objective in mind, the contents of the course are structured around three main parts:

- Part I - Introduction and General Design Considerations: this part of the course comprises two units that give a panoramic overview of the circuits and systems used in telecom applications, and explain their basic concepts and design issues like noise figure, linearity, low power and low-voltage operation.

- Part II - Analog/Digital Interfaces, which includes three units covering fundamentals and architectures of analog-to-digital and digital-to-analog converters for telecom systems.

- Part III - Signal Conditioning Circuits: this part, composed of three units, explains the main design issues and circuit techniques to implement amplifiers, filters, mixers, oscillators and frequency synthesizers used in the analog front-end of digital communication systems.

\section{B. Master Course}

The objective of the WT master course is to study the main wireless communication systems, putting especial emphasis on those important issues related to the design of RF CMOS integrated circuits, from the perspective of wireless transceivers. The fulfillment of this general objective is itemized in the following partial objectives:

- To analyze and to compare different architectures of digital radio transceivers.

- To study the main wireless communication standards and their application to the design of integrated circuits for communications.

- To learn the most important figures of merit and specifications required to characterize the performance of RF transceivers.

- To learn practical issues related to design methodologies and tools used to design and to synthesize RF transceivers.

The contents of WT master course are linked to the aforementioned objectives, and are organized in three blocks:

- Part I - Introduction: this part includes one unit that gives an overview of wireless communication systems, standards, trends and design challenges.
- $\quad$ Part II - Signal Processing Techniques and Wireless Communications: this part comprises two units. The first one deals with codification and modulation techniques, and the second one focuses on multiple access techniques and communication standards.

- $\quad$ Part III - RF Transceivers: Architectures and Design Considerations: this part is divided into two units and explains the most important transceiver architectures as well as practical issues related to the system planning and design methodologies.

\section{Proposed Methodology}

The courses described in previous section are taught by combining classical learning materials - like lecture notes, slides and practical/lab exercises - with multimedia resources and e-learning modules integrated in the WebCT virtual learning environment [3].

In addition to teach the fundamental principles of operation, circuit non-ideal deviations and practical design issues, the course contents are presented as parts of a systematic design procedure, where students can put the lessons learnt into practice, increasing their understanding about practical design problems explained during the course. The approach followed is based on the well-known top-down/bottom-up hierarchical synthesis methodology, in which a given system is divided into several abstraction levels, so that at each level, a design or sizing process takes place, by transmitting (or mapping) the system specifications in a hierarchical way - from the top level to the bottom level. The reverse path is followed to implement the hierarchical bottom-up verification process [4].

The simulation-based learning techniques carried out in this work are intended for telecom circuits and systems. To this end, three different hierarchy levels are defined: system level (transceiver), building-block level (low-noise amplifiers, mixers, oscillators, analog-to-digital converters, etc.), and circuit level (operational amplifiers, switches, capacitors, etc.). The course contents described in previous section are organized and structured around these hierarchy levels so that students can easily identify the different parts of telecom systems through the lessons they are learning.

In this context, traditional electrical (SPICE-like) simulators with transistor-level device models are not appropriate analysis and verification tools for several reasons. On the one hand, long CPU times - in the order of days or even weeks - may be required to evaluate the performance of some circuits and systems considered in classroom. On the other hand, as the model parameters, i.e. device sizes and biasing, are sometimes hierarchically far from system level, it is quite difficult for students to understand what is happening in the operation of the telecom systems under study, because the impact of a given transistor width or length has influence on a large number of performance metrics simultaneously. For instance, the size of differential input-pair MOS transistors in an Operational Transconductance Amplifier (OTA) may affect a number of electrical parameters, such as finite dc gain, output swing, input parasitic capacitance, unity gain-bandwidth product, etc. 


\section{A. Alternative Modeling and Simulation Methods}

An alternative method to transistor-level simulation approach that also runs on electrical simulators consists of using macromodels. Macromodels are usually implemented as equivalent circuits based on ideal voltage and current controlled sources, which allow to model main non-ideal effects in many different analog circuits. This well-known approach has the advantage that it can be combined with transistor-level implementation using the same simulator. Thus, critical parts of the system can be simulated at transistor-level whereas the remaining ones can be modeled less accurately by using macromodels. The latter technique is often referred to as multilevel simulation [4]. Obviously, the more parts of the system are simulated at transistor level, the slower the simulation will be, although in most practical cases, there is not a high improvement with respect to complete transistor-level simulations.

In this work, a simulation-based learning methodology has been followed that is based on the use of the so-called eventdriven behavioral simulation technique [4]. This technique has the following benefits for teaching and learning circuits and systems for communications. On the one hand, compared to the aforementioned simulation approaches, behavioral simulation achieves the best trade-off between accuracy and CPU time as illustrated in Fig. 1. On the other hand, students can analyze the fundamental operation of all circuit entities studied during the course, considering their main circuit non-ideal effects, but keeping a high abstraction model description. The key point in this methodology is to teach and learn how to describe those circuits by accurate behavioral equations that emulate their actual operation. In order to put this learning strategy into practice, the course contents are complemented with practical skills and tools in order to show students how they can obtain suitable behavioral models for the circuits they are studying in classroom. An efficient simulation platform is also used to evaluate the performance of telecom systems by using the behavioral models, which have been provided by the teacher or developed by the students. To this purpose, the procedure described below is followed in the course.

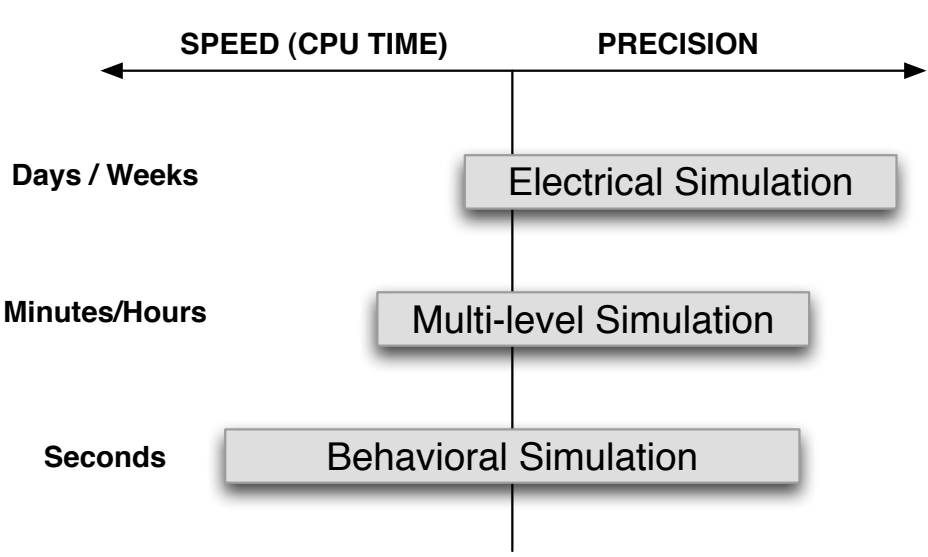

Figure 1. Comparison of different simulation approaches.

\section{B. From Circuit Analysis to Behavioral Equations}

The first step of the proposed methodology consists of explaining the circuits and systems, which are object of study in both EC and WT courses. This explanation involves both the ideal fundamental operation of the circuit as well as the definition and analysis of main non-ideal circuit effects. As a result of this analysis, closed-form equations describing the functionality of communication building blocks are derived as part of the course objectives. To this purpose, students can be trained to use algebra software like Mathematica [5] in order to minimize the amount of routine and handy calculations [6]. The final goal is that students can focus on understanding the circuit functionality and the impact of their physical non-ideal effects on telecom systems instead of getting lost in solving algebra problems. This analytical procedure allows students to derive precise equations that describe the functionality of building blocks studied during the course as a function of their main circuit error parameters. These equations constitute the basis for building accurate behavioral models to simulate complex systems made up of those building blocks.

As an example, let us consider the effect of the finite OTA dc gain on a Switched Capacitor (SC) Forward-Euler (FE) integrator like that shown in Fig. 2. This circuit is studied in Part II of the EC undergraduate course as an essential building block of ADCs for telecom systems. Students learn in classroom how to analyze circuits like this in the presence of different error mechanisms, analyzing both their isolate and cumulative effect. In the example of Fig. 2, the finite difference equation describing the behavioral of the circuit is given by:

$$
\begin{gathered}
v_{o}\left[n T_{s}\right]=\frac{1+\mu}{1+(1+g) \mu} v_{o}\left[(n-1) T_{s}\right]+ \\
+\frac{g}{1+(1+g) \mu} v_{i}\left[(n-1) T_{s}\right]
\end{gathered}
$$

where $v_{i}$ and $v_{o}$ are the input and output voltages of the integrator, respectively; $n T_{s}$ stands for the $n$th sampling time instant, with $T_{s}$ being the sampling period; $g=C_{s} / C_{i}$, $\mu \equiv 1 / A_{v}$ and $A_{v}$ stands for the finite OTA dc gain.

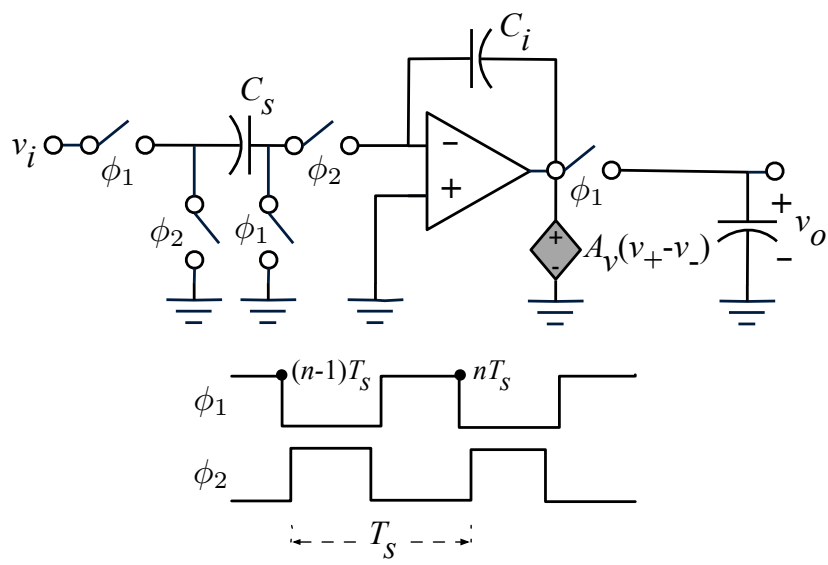

Figure 2. Example of circuit analyzed in classroom: an SC FE integrator. 


\section{From Behavioral Equations to Simulation Models}

Once students have learnt the principle of operation behind the different circuits and have analyzed their performance to obtain behavioral equations like that shown in (1), the next step is to implement suitable behavioral models based on those equations. These models and the corresponding simulation engine can be codified in a number of platforms using different programming/modeling languages like $\mathrm{C}$, standard Hardware Description Languages (HDL) such as VHDL or Verilog, etc. In this work, behavioral models used in classroom have been implemented in the MATLAB/SIMULINK environment. This well-known mathematical package - which constitutes a standard CAD platform today in science and engineering presents a number of advantages for students in terms of friendliness of the user interface; flexibility for the extension of new circuit/system elements and a huge number of tools for signal post-processing [7].

Different model implementation approaches can be followed: one is based on the interconnection of SIMULINK standard library blocks. The resulting block diagrams are very intuitive for students to evaluate communication systems with simple models, at the price of increasing the CPU time and reducing the accuracy. The second modeling approach consists of using the so-called SIMULINK $S$-functions, which allows to simulate more complex circuit and physical-level phenomena in an efficient way, although they are not so intuitive for students and require to learn some basic skills on computer languages such as $\mathrm{C}$ [7]. For that reason, this behavioral modeling strategy is explained in classroom, although the most complex models are not developed by students. They learn the way to do it and sometimes can develop some basic models.

Fig. 3 shows the step-by-step procedure required to implement a behavioral model in the SIMULINK environment by using $\mathrm{C}$-coded $\mathrm{S}$-functions. The main steps are [8]:

- Definition of a computational model, based on the behavioral equations analyzed in classroom.

- Generation of the $C$ code corresponding to the computational model defined in the previous step.

- Implementation of the computation model into a $C$ MEX S-function. To this purpose, SIMULINK provides different $\mathrm{S}$-function template files, which can accommodate the C-coded computation model generated in previous step.

- Compilation of the S-function. This is done by using the mex utility provided by MATLAB [7]. The resulting object files are dynamically compiled and linked in SIMULINK when needed in a given simulation.

- Incorporation of the model into the SIMULINK environment. This can be done by using the $\mathrm{S}-$ function block of the SIMULINK libraries [7]. A S-function block diagram is created including input/output pins, model parameters, etc.

As an illustration, Fig. 4 shows the main parts of the SIMULINK model used for a SC FE integrator like that shown in Fig. 2. The associated S-function name and the model parameters are entered into the Block Parameter dialogue box. The input/output terminal ports are connected to the Sfunction block in the SIMULINK block diagram. In order to make the use of S-functions easy in more complex systems, a Block Mask can be created. An specific Mask Icon can be used instead of the SIMULINK subsystems standard icon. This is more intuitive for students since they can better distinguish the different building blocks in a telecom system.

\section{EXAMPLES AND CASE STUDIES}

Every academic year, at least one case study is linked to both the second and the third parts of the courses described in Section II. This allows students to put theory into practice by applying the proposed simulation-based learning methodology. As an application, this section shows two case studies, which have been used for several years. The first one - linked to Part II of the EC undergraduate course - consists of the high-level design of $\Sigma \Delta$ Modulators ( $\Sigma \Delta \mathrm{Ms})$ for telecom applications. The second case study - given in Part III of the WT master course deals with the system planning and simulation of Direct Conversion Receivers (DCRs).

\section{A. Case Study 1: Modeling and Simulation of $\Sigma \Delta M s$}

The EC course includes several case studies that aim to teach students how to use behavioral modeling and simulation techniques to analyze the effect of circuit errors on the performance of ADCs, in order to map system-level specifications onto building-block specifications in a high-level

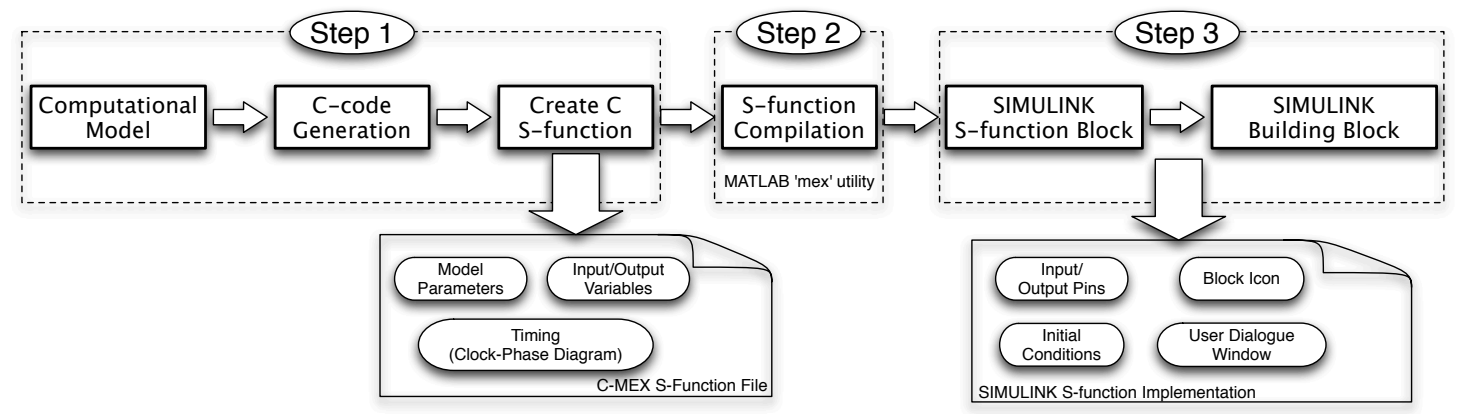

Figure 3. Step-by-step procedure to incorporate a behavioral model into the MATLAB/SIMULINK environment using C-MEX S-functions. 
sizing process. As stated above, different approaches for the implementation of behavioral models of both pipeline [9] and $\Sigma \Delta$ ADCs [8], [10] have been considered in the course.

As an illustration, Fig. 5 shows the block diagram of a 2ndorder $\Sigma \Delta \mathrm{M}$ implemented using behavioral models based on $\mathrm{S}$ functions as proposed in [8]. Behavioral modeling techniques are used here with a twofold objective. On the one hand, students learn fundamental concepts of ADCs and how to obtain their main performance metrics like Fast Fourier Transform (FFT), harmonic distortion, Signal-to-Noise Ratio (SNR), etc. On the other hand, students can easily understand the principle of operation of basic circuits - like the SC FE integrator in Fig. 2 - as well as the influence of their most important circuit limiting factors. Moreover, they can learn how to model nonideal effects in order to analyze complex circuits and systems, studying both their isolate effect and their cumulative effect.

\section{B. Case Study 2: Modeling and Simulation of DCRs}

The main purpose of this case study is to learn how to analyze and to simulate wireless transceivers at the system level, but taking into consideration circuit-level effects. To this purpose, a DCR is simulated in SIMULINK environment using the behavioral model available in the SIMULINK block set presented in [11]. This block set includes a SIMULINK library with the main RF circuit models that are needed to implement wireless transceivers, namely: low-noise amplifiers, mixers, oscillators, filters and programmable amplifiers. There is also a library including other building blocks like the antenna, the duplexer filter and RF switches, which are also required to implement reconfigurable architectures.

Behavioral models of building blocks include the main ideal functionality as well as their associated Noise Figure (NF) and non-linearity - modeled by the input-referred second- and third-order intercept points. In addition to these general parameters, some block-specific errors have also been included, like oscillator phase noise and mixer offset [11]. As an illustration, Fig. 6 shows the implementation of the DCR in SIMULINK, highlighting some parts of the behavioral models.

In order to help students with the use of the mentioned SIMULINK block set, several MATLAB scripts are available in the WebCT site of the course. Some of these scripts are used to set up receiver parameters, like gain, noise figure,

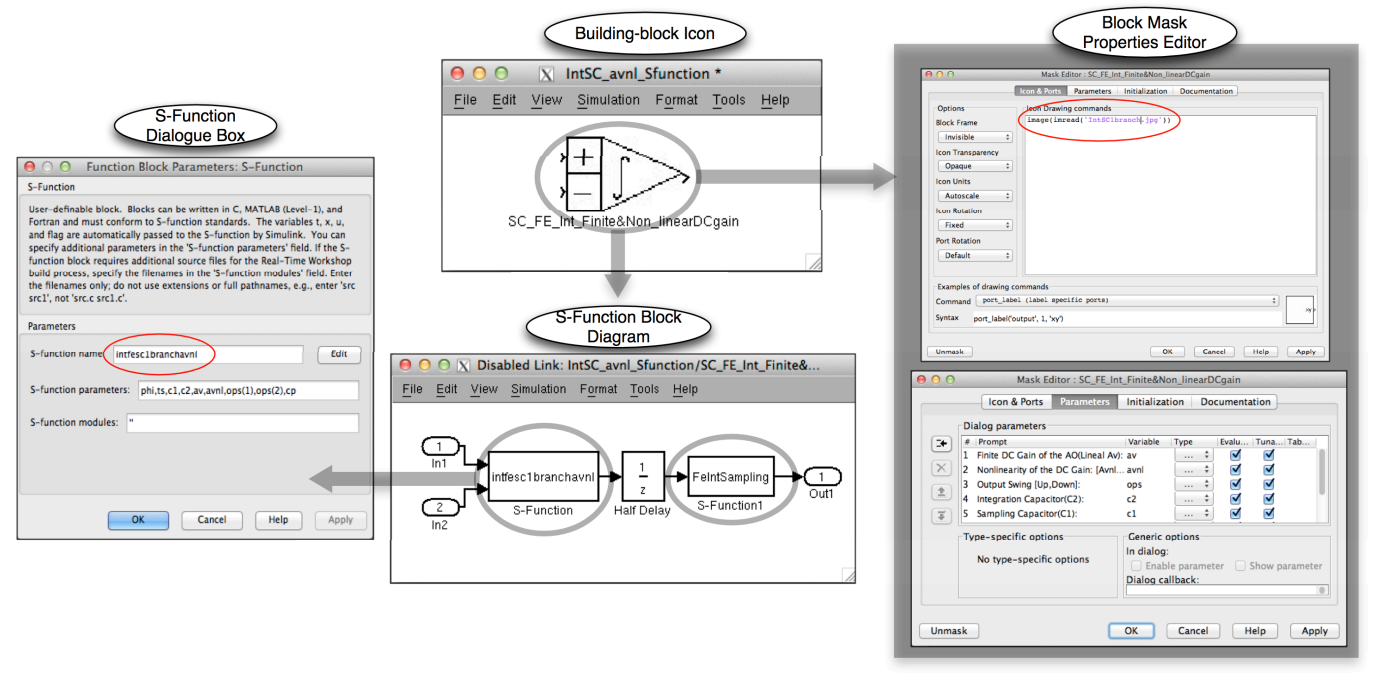

Figure 4. Illustrating the SIMULINK S-function model implementation of the SC FE integrator shown in Fig. 2.

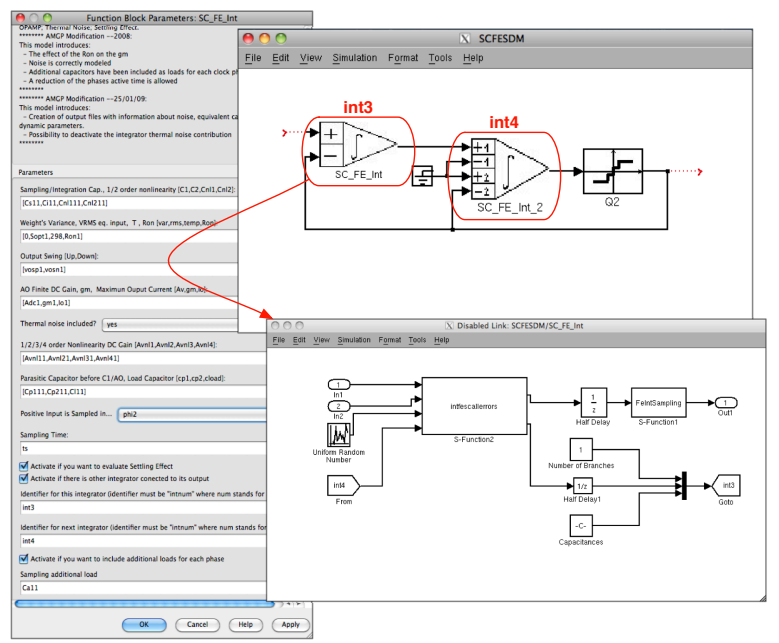

Figure 5. Illustrating some behavioral models used to simulate $\Sigma \Delta \mathrm{Ms}$.

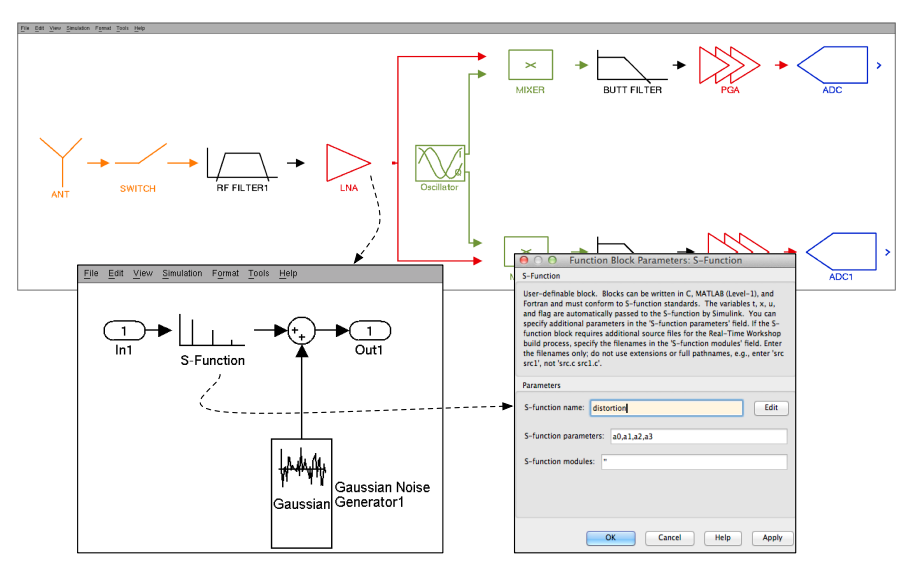

Figure 6. Illustrating some behavioral models used to simulate DCRs. 
intermodulation distortion intercept points, etc. Other scripts are used to simulate performance metrics like NF, output spectra and level diagrams. This way, the explanation of the behavioral models and MATLAB scripts in classroom provides students with complementary skills that may be quite useful for their professional career, since they may follow similar approaches to model other complex systems.

\section{STUDENTS' FEEDBACK}

Students attending the EC course were surveyed during four consecutive academic years in order to give us their feedback to improve the quality of the course in terms of its contents, learning resources and the pedagogic procedure. The number of students participating in this survey varied every year, being an average of 50 participants approximately. Fig. 7 (a) shows the interest and motivation demonstrated by the students for those course contents taught by using the proposed simulation-based methodology. Note that a huge majority of students appreciated the use of behavioral simulation as a learning resource, increasing year after year (except for the third year surveyed) and more than $90 \%$ of them become interested in the proposed methodology in the last year.

Students were asked about their level of satisfaction with the simulation-based methodology, assigning a grade from 1 to 4 , with 1 being "bad" and 4 being "very good". As illustrated in Fig. 7(b), students were roughly well satisfied, especially in the last years, when the proposed simulated-based methodology became more mature and refined. Indeed, the highest level of satisfaction was obtained in the last year, when more than $50 \%$ of students expressed the maximum level of satisfaction.

Finally, Fig. 7(c) shows the overall impression shown by students. It is clear that a vast majority of students showed a good impression of the course, becoming better in the last two years, and particularly in the last one, when almost all students considered the course either "good" ( 70\%) or "very good" $(\sim 30 \%)$.

\section{CONCLUSIONS}

Behavioral modeling and simulation techniques have been presented as a teaching methodology used in different courses dealing with circuits and systems for wireless telecom. Based on more than ten years experience, it has been demonstrated to be a method well appreciated by the majority of students, who prefer the course contents including simulation-based learning resources to those taught using just classical methods.

\section{REFERENCES}

[1] A. Stefan et al., "Behavioral Models for Microwave Circuit Optimization," Proc. of IEEE Global Engineering Education Conference (EDUCON), pp. 806-808, 2011.

[2] A. Abramovitz, "Teaching Behavioral Modeling and Simulation Techniques for Power Electronics Courses," IEEE Trans. on Education, vol. 54, pp. 523-530, November 2011.

[3] D. Pishva et al.,"A Survey on HowBlackboard is Assisting Educational Institutions Around the World and the Future Trends," Proc. of IEEE Int. Conf. on Advanced Communication Technology (ICACT), pp. 15391543, 2010.
[4] G. Gielen and J. Franca, "CAD Tools for Data Converter Design: An Overview," IEEE Trans. on Circuits and Systems II: Analog and Digital Signal Processing, vol. 43, pp. 77-89, February 1996.

[5] Wolfram, "Mathematica," Wolfram Research., 2011.

[6] J. Kyncl and M. Novotny, "Education of Digital and Analog Circuits Supported by Computer Algebra System," Proc. of IEEE Int. Symposium on Circuits and Systems (ISCAS), pp. 341-344, 2011.

[7] Mathworks, MATLAB R2011b Documentation. [Online]. Available: http://www.mathworks.com, 2011.

[8] J. Ruiz-Amaya et al., "High-Level Synthesis of Switched-Capacitor, Switched-Current and Continuous-Time $\Sigma \Delta$ Modulators Using SIMULINK-based Time-Domain Behavioral Models," IEEE Trans. on Circuits and Systems - I: Regular Papers, vol. 51, pp. 1795-1810, September 2005.

[9] J. Ruiz-Amaya et al., "Behavioral Modeling Simulation and High-Level Synthesis of Pipeline A/D Converters," Proc. of IEEE Int. Symposium on Circuits and Systems (ISCAS), pp. 5609-5612, 2005.

[10] P. Malcovati et al., "Behavioral modeling of switched-capacitor sigmadelta modulators," IEEE Trans. on Circuits and Systems - I: Regular Papers, vol. 50, pp. 352-364, March 2003.

[11] A. Morgado et al., "A SIMULINK Block Set for the High-Level Simulation of Multistandard Radio Receivers," Proc. of IEEE Int. Symposium on Circuits and Systems (ISCAS), pp. 2950-2953, 2007.

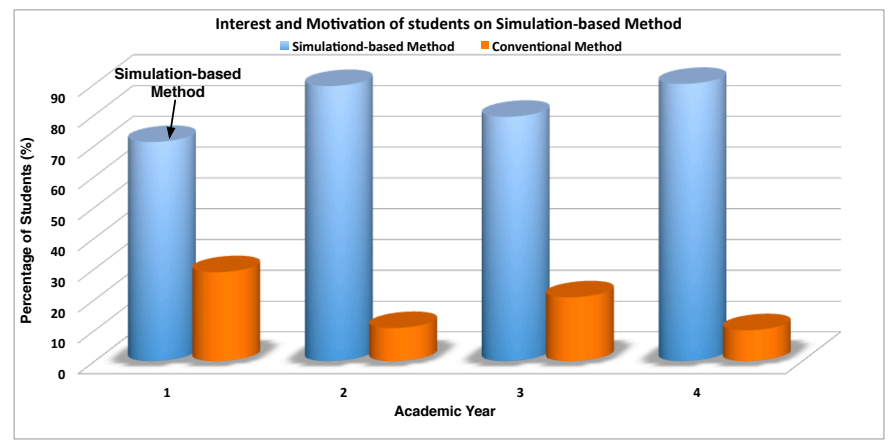

(a)

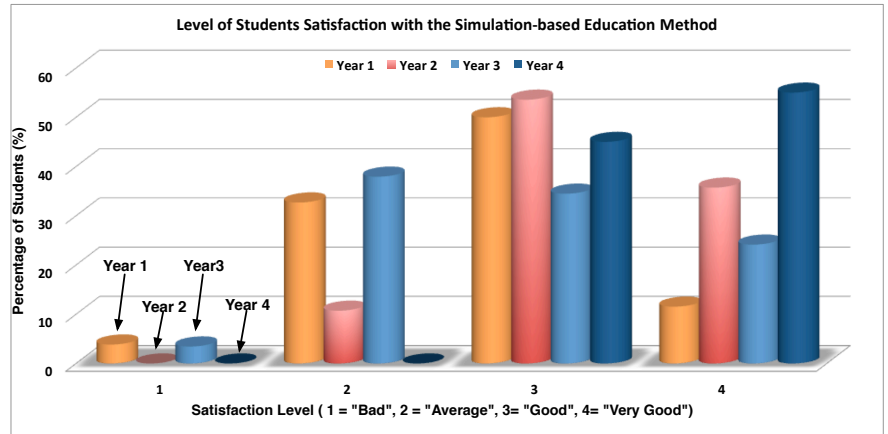

(b)

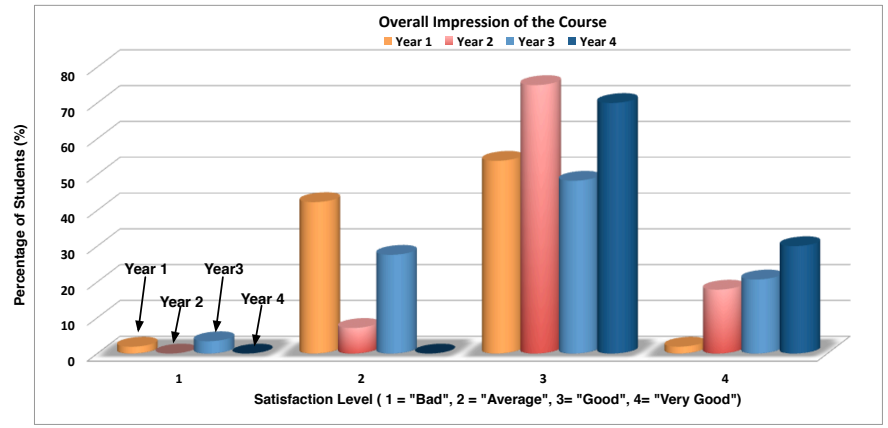

(c)

Figure 7. Students' feedback and results: (a) Interest and motivation for simulation-based contents. (b) Satisfaction level. (c) Overall impression. 